\title{
Selective Control over Fragmentation Reactions in Polyatomic Molecules Using Impulsive Laser Alignment
}

\author{
Xinhua Xie, ${ }^{1}$ Katharina Doblhoff-Dier, ${ }^{2,3}$ Huailiang Xu ${ }^{1,4}$ Stefan Roither, ${ }^{1}$ Markus S. Schöffler, ${ }^{1}$ Daniil Kartashov, ${ }^{1}$ \\ Sonia Erattupuzha, ${ }^{1}$ Tim Rathje, ${ }^{5}$ Gerhard G. Paulus, ${ }^{5,6}$ Kaoru Yamanouchi, ${ }^{7}$ Andrius Baltuška, ${ }^{1}$ \\ Stefanie Gräfe, ${ }^{3,8}$ and Markus Kitzler ${ }^{1, *}$ \\ ${ }^{1}$ Photonics Institute, Vienna University of Technology, A-1040 Vienna, Austria \\ ${ }^{2}$ Institute for Theoretical Physics, Vienna University of Technology, A-1040 Vienna, Austria \\ ${ }^{3}$ Institute for Physical Chemistry, Friedrich-Schiller University Jena, D-07743 Jena, Germany \\ ${ }^{4}$ State Key Laboratory on Integrated Optoelectronics, College of Electronic Science and Engineering, Jilin University, \\ Changchun 130012, China \\ ${ }^{5}$ Institute of Optics and Quantum Electronics, Friedrich-Schiller-University Jena, D-07743 Jena, Germany \\ ${ }^{6}$ Helmholtz Institute Jena, D-07743 Jena, Germany \\ ${ }^{7}$ Department of Chemistry, School of Science, The University of Tokyo, Tokyo 113-0033, Japan \\ ${ }^{8}$ Abbe Center of Photonics, Friedrich-Schiller-University Jena, D-07743 Jena, Germany
}

(Received 29 January 2014; published 25 April 2014)

\begin{abstract}
We investigate the possibility of using molecular alignment for controlling the relative probability of individual reaction pathways in polyatomic molecules initiated by electronic processes on the few-femtosecond time scale. Using acetylene as an example, it is shown that aligning the molecular axis with respect to the polarization direction of the ionizing laser pulse does not only allow us to enhance or suppress the overall fragmentation yield of a certain fragmentation channel but, more importantly, to determine the relative probability of individual reaction pathways starting from the same parent molecular ion. We show that the achieved control over dissociation or isomerization pathways along specific nuclear degrees of freedom is based on a controlled population of associated excited dissociative electronic states in the molecular ion due to relatively enhanced ionization contributions from inner valence orbitals.
\end{abstract}

DOI: 10.1103/PhysRevLett.112.163003

PACS numbers: $33.80 . \mathrm{Rv}, 42.50 . \mathrm{Hz}, 82.50 . \mathrm{Nd}$

Control over the photodissociation dynamics of molecules, an important goal in the physical, chemical, and biological sciences, has been successfully demonstrated using coherent control schemes that apply weak (shaped) femtosecond laser pulses to resonantly excite vibrational dynamics (see, e.g., [1] for a review). Using the carrierenvelope phase (CEP) of intense, few-cycle laser pulses as the control parameter, a new nonresonant paradigm of dissociation control has emerged. In this scheme, control over the timing of field oscillations of the laser pulses rather than only their envelopes and/or frequency evolution allows for dissociation control on the time scale of the intramolecular electron dynamics. This has been demonstrated for fragmentation processes starting from ionic states of different diatomic and triatomic molecules [2-6]. Very recently, we reported an extension of CEP control to polyatomic molecules with their much more complex multielectronic structure [7]. It has been shown that a CEP-controlled initiation or suppression of molecular fragmentation can be achieved via the selective removal of electrons from specific valence states by tuning the recollision energy with the CEP. Controllability in this scheme, however, is limited to laser parameters for which recollision-ionization is the dominant ionization mechanism. In addition, this mechanism does not in general permit selective control over individual fragmentation channels.

In this Letter, using the acetylene molecule, $\mathrm{C}_{2} \mathrm{H}_{2}$, as an example, we demonstrate that the orientation of the molecular axis relative to the laser polarization direction can serve as a control tool to determine not only the overall fragmentation yield but also the relative probability of individual reaction pathways in polyatomic molecules over a wide range of laser peak intensities and pulse durations. The method builds on the alignment-sensitive ionization yields from inner and outer valence orbitals, previously reported for small molecules such as $\mathrm{O}_{2}, \mathrm{~N}_{2}$, and $\mathrm{CO}_{2}$ [8-13], and exploits the fact that selective ionization from specific inner valence orbitals allows for the controlled population of electronic surfaces in the ion associated with certain reaction pathways. As a consequence, we are for the first time able to demonstrate channel-selective fragmentation control in a polyatomic molecule using a strong-field nonresonant scheme.

In our experiments, we impulsively align [14] $\mathrm{C}_{2} \mathrm{H}_{2}$ molecules in an ultrahigh vacuum chamber $\left(1.3 \times 10^{-10} \mathrm{mbar}\right)$ using a linearly polarized, nonionizing (intensity $\approx 2 \times 10^{13} \mathrm{~W} / \mathrm{cm}^{2}$ ) temporally stretched (to $\approx 50 \mathrm{fs}$ ) laser pulse split from the output of a 5-kHz titanium-sapphire 
laser amplifier system. The remaining portion of the laser output, compressed to a sub-5 fs (full width at half maximum) duration, serves as the probe pulse. The duration and the CEP of each probe pulse is measured on a single-shot basis by a stereo-above-threshold ionization phase meter [15]. The probe pulse, collinearly polarized with the pump pulse and delayed relative to it by $\Delta t$, is sufficiently strong to (multiply) ionize the $\mathrm{C}_{2} \mathrm{H}_{2}$ molecules. The momenta of the resulting ions are detected in coincidence [16-18], which allows selecting and examining individual fragmentation channels. The peak intensity of the probe laser pulse is determined from calibration measurements using circularly polarized light [19].

Upon interaction with the alignment pulse, a rotational wave packet is created in the neutral $\mathrm{C}_{2} \mathrm{H}_{2}$ molecule. By following an alignment-sensitive signal-for example the yield of protons ejected from the molecule after application of the strong probe pulse [Fig. 1(a)]—over $\Delta t$, the delays
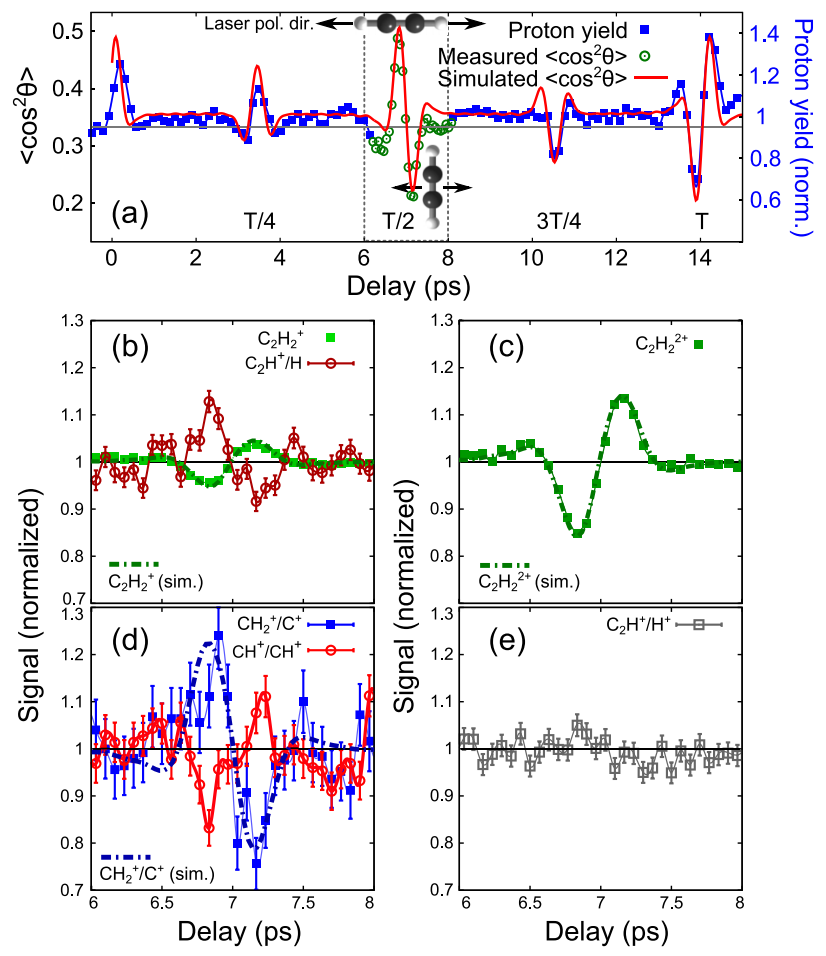

FIG. 1 (color online). (a) Measured (green circles) and simulated (red line) $\left\langle\cos ^{2}(\theta)\right\rangle$ as a function of delay between the alignment pulse and the probe pulse with a peak intensity of $4 \times 10^{14} \mathrm{~W} / \mathrm{cm}^{2}$, with $\theta$ the angle of the molecular axis relative to the polarization direction of the alignment pulse. The blue squares are the normalized measured yield of protons ejected from $\mathrm{C}_{2} \mathrm{H}_{2}$ over pump-probe delay (right axis applies). (b-e) Measured yields (normalized) of all detected ionization and fragmentation channels over pump-probe delay around the half revival. (b) $\mathrm{C}_{2} \mathrm{H}_{2}^{+}$(green squares), $\mathrm{C}_{2} \mathrm{H}^{+} / \mathrm{H}$ (dark red circles), (c) $\mathrm{C}_{2} \mathrm{H}_{2}^{2+}$ (green squares), (d) $\mathrm{CH}_{2}^{+} / \mathrm{C}^{+}$(blue squares), $\mathrm{CH}^{+} / \mathrm{CH}^{+}$(red circles), (e) $\mathrm{C}_{2} \mathrm{H}^{+} / \mathrm{H}^{+}$(gray squares). Dashed-dotted lines represent the results of simulations detailed in the text for $\mathrm{C}_{2} \mathrm{H}_{2}^{+}$(b), $\mathrm{C}_{2} \mathrm{H}_{2}^{2+}$ (c), and $\mathrm{CH}_{2}^{+} / \mathrm{C}^{+}$(d). for the quarter, half, and full revival of this wave packet can be identified by the modulations of this signal. The alignment quality achieved in our experiment at the half revival $(\Delta t \approx 7 \mathrm{ps})$ is quantified by deriving the value of $\left\langle\cos ^{2}(\theta)\right\rangle$ from a four-body coincidence measurement of ions created by Coulomb explosion of $\mathrm{C}_{2} \mathrm{H}_{2}^{4+}$ [green circles in Fig. 1(a)]. For these data, about $6 \times 10^{4}$ four-body coincidence hits were registered at an approximate rate of $3 \times 10^{-4}$ per laser shot. Both series of experimental data are well reproduced by simulations that compute the dynamics of a rotational wave packet [20] induced by a laser pulse with the same parameters as in the experiment using a polarizability orthogonal to the molecular axis of $2.7 \AA^{3}$ and an anisotropy of $1.8 \AA^{3}$ [21], a rotational constant of $1.18 \mathrm{~cm}^{-1}$ [22], and an initial rotational temperature of $100 \mathrm{~K}$ equal to the one estimated from the experiment [red line in Fig. 1(a)].

The measured yields of the four identified fragmentation channels, as well as the yields of the cation and the dication, depend crucially on $\Delta t$, as shown in Figs. 1(b)-1(e) near the half revival for a probe pulse intensity of $4 \times 10^{14} \mathrm{~W} / \mathrm{cm}^{2}$ and a sub-5 fs duration. During the half revival, the relative alignment of the molecule to the laser polarization direction changes from preferentially parallel at $6.8 \mathrm{ps}$ to preferentially orthogonal at $7.2 \mathrm{ps}$. Hence, the fragmentation yield of most channels critically depends on the relative alignment of the molecule to the laser polarization direction. By contrast, no dependence of the fragmentation yields on the CEP was observed. An important feature of the measured fragmentation yields is that their delay-dependence differs for various examined fragmentation channels. While, for example, the yield of the fragmentation channel $\mathrm{C}_{2} \mathrm{H}_{2}^{+} \rightarrow \mathrm{CH}_{2}^{+}+\mathrm{C}^{+}$peaks at parallel alignment, the yield of the channel $\mathrm{C}_{2} \mathrm{H}_{2}^{+} \rightarrow \mathrm{CH}^{+}+$ $\mathrm{CH}^{+}$peaks at perpendicular alignment [Fig. 1(d)]. By adjustment of $\Delta t$, it is thus possible to relatively enhance the yield of one channel with respect to the other (in our experiment by about $150 \%$ ). This finding clearly demonstrates the potential of molecular alignment to selectively enhance or suppress individual fragmentation channels of the same parent ion of polyatomic molecules on a very short time scale. The possibility of channel-selective control is a novel feature as compared to previous attempts of controlling the fragmentation behavior of polyatomic molecules [7], where only the overall fragmentation yield, but not the relative yields of individual fragmentation channels, could be controlled.

We now show that the control gained over the fragmentation yields in the experiment (Fig. 1) is based on different angular-dependent ionization rates of inner and outer valence electrons. As has been shown in the literature (see, e.g., [23-32]), depending on the symmetry of the orbital and its relative orientation to the direction of the laser electric field, ionization rates differ substantially. While, qualitatively, $\sigma$-type orbitals are in general preferentially field ionized 
when the laser polarization direction is parallel to the molecular axis, $\pi$-type orbitals are preferentially ionized for perpendicular alignment. Quantitatively, however, ionization rates depend on the shapes, symmetries, ionization potentials, and multielectron nature of the molecular orbitals, as well as on the parameters of the ionizing laser pulse. To analyze the ionization behavior underlying the experimental observations in Fig. 1, we have numerically calculated the angle-dependent field-ionization yield by time-dependent density functional theory (TDDFT) using the остOPUS code [33]. Acetylene and its cation were subjected to a linearly polarized Gaussian pulse with a wavelength of $800 \mathrm{~nm}$, a duration of $4.5 \mathrm{fs}$, and a peak intensity of $2.8 \times 10^{14} \mathrm{~W} / \mathrm{cm}^{2}$. The slightly lower intensity was chosen to avoid space charge effects due to ionization of more than one electron and to avoid significant depletion of molecular orbitals since both could lead to an inaccurate description by the TDDFT method. The ionization probabilities from the $\pi$ and $\sigma$ orbitals of the neutral and the cation, shown for the neutral in Fig. 2(e), were calculated from the norms of the respective orbitals in the neutral and the cation before and long after the laser pulse. Double-ionization yields were calculated assuming sequential ionization. Figures 2(a)-2(d) display the calculated angular-dependent ionization probabilities from selected orbitals of acetylene and the acetylene cation, respectively. Figure 2(a) shows that single ionization from the $\pi$ orbitals (HOMO) features only a weak
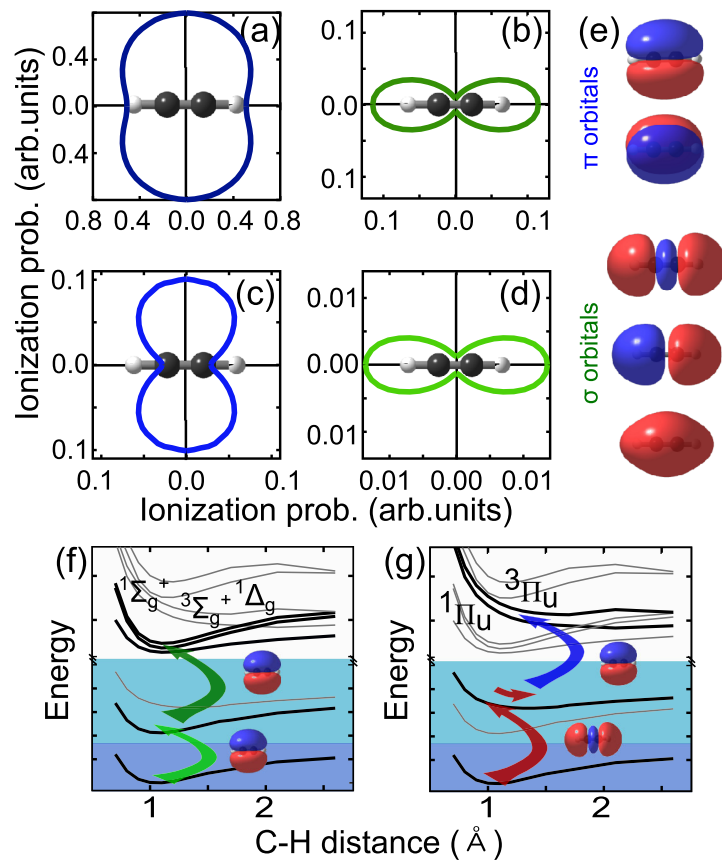

FIG. 2 (color online). Angular-dependent ionization probabilities for different valence orbitals calculated by TDDFT: (a) $\pi$ orbitals of $\mathrm{C}_{2} \mathrm{H}_{2}$, (b) $\sigma$ orbitals of $\mathrm{C}_{2} \mathrm{H}_{2}$, (c) $\pi$ orbitals of $\mathrm{C}_{2} \mathrm{H}_{2}^{+}$, (d) $\sigma$ orbitals of $\mathrm{C}_{2} \mathrm{H}_{2}^{+}$. (e) Schematics of the valence orbitals of $\mathrm{C}_{2} \mathrm{H}_{2}$. (f) Proposed ionization scheme for $\mathrm{C}_{2} \mathrm{H}_{2}$ to end in the dication, $\mathrm{C}_{2} \mathrm{H}_{2}^{2+}$, or $(\mathrm{g})$ the isomerization channel, $\mathrm{CH}_{2}^{+} / \mathrm{C}^{+}$. dependence on the orientation of the molecule with respect to the laser polarization direction since at this field strength the ionization proceeds almost over the barrier. In contrast, ionization from the lower lying $\sigma$ orbitals [Fig. 2(b)] and from the cationic orbitals [Figs. 2(c) and 2(d)] features a strong dependence on the angle between the laser polarization direction and the internuclear axis.

Ionization from different electronic states will result in the population of different electronic states in the dication: If two $\pi$ electrons are removed, the stable electronic ground state of the dication is reached, and dissociation is inhibited. The removal of one $\pi$ and one $\sigma$ electron, however, puts the dication into an excited electronic state. Different electronic states feature, in general, different characteristic potential energy surfaces. The dissociation or isomerization processes that follow the ionization event may therefore, in turn, proceed along different specific nuclear degrees of freedom and as a result will end up in different fragmentation channels. Controlling the population of a specific excited electronic surface in the dication by determining the ratio of ionization from different orbitals (e.g., $\sigma$ vs $\pi$ ) using the molecular alignment as a knob, allows us, thus, to control the yield of a certain fragmentation channel associated with this electronic surface. This is the essence of the control method introduced here.

The simplest cross-check for the applicability of this control scheme is to test it on the experimentally observed yield modulation of the nondissociating dication, $\mathrm{C}_{2} \mathrm{H}_{2}^{2+}$. The three lowest excited states of $\mathrm{C}_{2} \mathrm{H}_{2}^{2+}\left({ }^{1} \Sigma_{g}^{+},{ }^{3} \Sigma_{g}^{+},{ }^{1} \Delta_{g}\right)$, all reached by removing two electrons from the $\pi$-electron system, are strongly bound. The next higher lying states $\left({ }^{1} \Pi_{u},{ }^{3} \Pi_{u}\right)$, reached by removal of a $\pi$ and a $\sigma$ electron are dissociative. At the laser peak intensity used for the data in Fig. 1, sequential ionization is the dominant mechanism to produce $\mathrm{C}_{2} \mathrm{H}_{2}^{2+}$ [34]. As a consequence, we expect the yield of $\mathrm{C}_{2} \mathrm{H}_{2}^{2+}$ to follow the alignment dependence of the probability for sequentially removing two $\pi$ electrons [see the sketch in Fig. 2(f)]. Indeed, convolution of the alignment-dependent ionization rate from the $\sigma$ orbitals calculated by TDDFT with the simulated time-dependent alignment distribution quantitatively reproduces the experimental data to a high degree [see Fig. 1(c)]. Similarly, the measured yield of $\mathrm{C}_{2} \mathrm{H}_{2}^{+}$as a function of $\Delta t$ very closely follows the predictions for the removal of a single $\pi$ electron. The modulation depth of the yield over $\Delta t$ is less pronounced in this case due to the weak alignment dependence of ionization from the HOMO at this intensity, as discussed above [cf. Fig. 2(a)].

We now extend our discussion to the measured alignmentsensitive yields of the individual fragmentation channels from the dication [Figs. 1(d) and 1(e)]. Our concern is to understand the connection between the experimentally observed yield modulations and the alignment-dependent ionization dynamics in order to investigate the generality of using the molecular alignment for reaction control. We start 
with the observed yield modulation of the proton-migration channel with final products $\mathrm{CH}_{2}^{+} / \mathrm{C}^{+}$, which features a maximum for parallel alignment. Intuitively, this suggests that the removal of a $\sigma$-type electron plays a significant role. Indeed, the measured yield modulation can very well be reproduced by our simulations assuming the sequential removal of a $\sigma$-type and a $\pi$-type electron [see Fig. 1(d)]. Such a double-ionization dynamics leads to the population of the ${ }^{1} \Pi_{u}$ or ${ }^{3} \Pi_{u}$ states, which are known to have a low reaction barrier for acetylene-vinylidene isomerization [35] and can thus lead to the production of $\mathrm{C}^{+}$. The corresponding scenario for the dissociation reaction following the ultrafast isomerization dynamics associated with the two ionization events is sketched in Fig. 2(g). Because of the high alignment sensitivity of ionization from the $\sigma$ orbitals, with a better quality of alignment than the one achieved in our experiment, a still higher degree of experimental control over the yield of the $\mathrm{CH}_{2}^{+} / \mathrm{C}^{+}$channel can be achieved.

As outlined above, a particular strength of using molecular alignment for controlling the yield of certain fragmentation channels is that it allows us to distinguish between different molecular pathways and to increase or decrease their relative importance. As shown in Fig. 1(d), the isomerization channel $\mathrm{CH}_{2}^{+} / \mathrm{C}^{+}$can be distinguished from the $\mathrm{C}-\mathrm{C}$ bond breaking channel $\mathrm{CH}^{+} / \mathrm{CH}^{+}$by their opposite alignment sensitivity. This suggests that the dissociation along the $\mathrm{C}-\mathrm{C}$ bond is initiated by the removal of at least one $\pi$ electron. Indeed, this is what our theoretical considerations indicate. A detailed description of the ionization process at the parameters of the experiment, however, turns out to be a challenge, as the double-ionization dynamics very likely takes place not only sequentially but to a certain extent also by difficult to model recollisional ionization, resulting in the population of potentially highly excited electronic states in the dication.

Finally, we discuss the third observed fragmentation channel consisting in a deprotonation reaction with final products $\mathrm{C}_{2} \mathrm{H}^{+} / \mathrm{H}^{+}$, whose yield does not show any significant modulation with $\Delta t$ [Fig. 1(e)]. To understand this insensitivity to the alignment, we first note that the KER distribution of the fragments features a clear doublepeak structure [Fig. 3(a)]. This indicates the presence of (at least) two competing reaction pathways. By separating the events for low and high KER, it becomes apparent that the two reaction pathways have opposite alignment dependence [Fig. 3(b)]: While the low KER part follows the predictions for ionization from one $\pi$ and one $\sigma$ orbital, the high KER part follows the predictions for electron removal from the $\pi$ system only. Only in the KER-integrated picture, where the two pathways contribute with nearly equal probability, the alignment dependence of the overall yield vanishes. Is it possible, in addition to controlling the relative importance of selected channels by the alignment, to also control the reaction pathway using additional knobs? We have experimentally tested this possibility by varying the peak intensity, duration, and polarization state
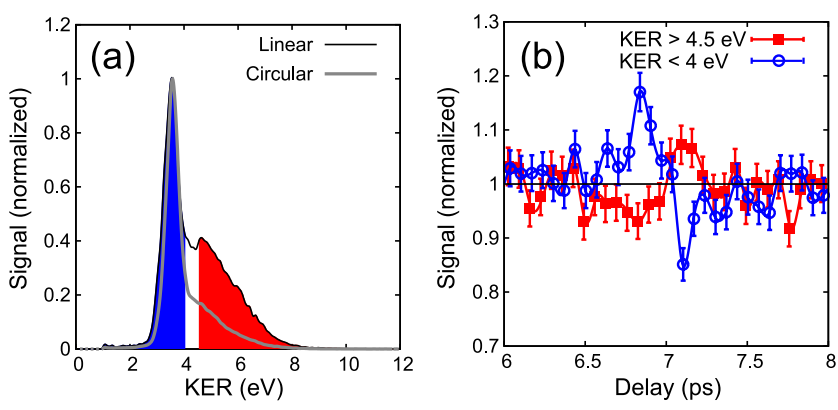

FIG. 3 (color online). (a) Measured KER spectra of channel $\mathrm{C}_{2} \mathrm{H}^{+} / \mathrm{H}^{+}$for linearly (black line) and circularly (gray line) polarized pulses with intensities $4 \times 10^{14}$ and $3 \times 10^{14} \mathrm{~W} / \mathrm{cm}^{2}$, respectively. (b) Yield over pump-probe delay for pairs of fragments with KER $\leq 4 \mathrm{eV}$ (blue circles) and KER $\geq 4.5 \mathrm{eV}$ (red squares), measured with linearly polarized probe pulses.

of the ionizing laser pulse. Our experimental results show that the relative contribution of the high-energy fragments $(\mathrm{KER} \geq 5 \mathrm{eV})$ decreases substantially for circular polarization [Fig. 3(a)], higher intensities $\left(I \approx 7 \times 10^{14} \mathrm{~W} / \mathrm{cm}^{2}\right)$, or longer pulses (we tested positively chirped pulses with durations up to $18 \mathrm{fs}$ and the suppression saturates at about $10 \mathrm{fs})$. From that we conclude that nonsequential processes, such as recollision-ionization and recollision-excitation, may play a non-negligible role for events in the high KER peak, while events in the low KER peak dominantly originate from sequential ionization. Molecular alignment in combination with fine-tuned laser parameters, thus, does not only allow us to determine the relative yield of individual channels but even the molecular pathway toward a certain final set of fragment ions.

Until now we have focused only on fragmentation reactions starting from the dication. The control scheme is, however, also applicable to the control of dissociative channels from the cation, where the molecule splits into a charged and a neutral fragment. We demonstrate this using the dissociation reaction $\mathrm{C}_{2} \mathrm{H}_{2}^{+} \rightarrow \mathrm{C}_{2} \mathrm{H}^{+}+\mathrm{H}$ as an example. The measured probability of this dissociation reaction is maximum for parallel and minimum for perpendicular alignment - opposite to the alignment dependence of $\mathrm{C}_{2} \mathrm{H}_{2}^{+}$ [Fig. 1(b)], which indicates that this channel originates dominantly from electronic states reached by removing an electron from $\sigma$ orbitals. Simulations that assume ionization from the highest lying $\sigma$ orbital, as also proposed in the literature [36], however, overestimate the experimentally observed modulation depth by a factor of 2 . This suggests that also other possible pathways may exist for this channel, for example, dissociation due to field excitation from the cationic ground state to the ${ }^{2} \Pi_{g}$ or ${ }^{2} \Sigma_{g}^{+}$state after the single ionization event. Independent of the actual dissociation dynamics, just as the opposite alignment dependence of the $\mathrm{CH}^{+} / \mathrm{CH}^{+}$and $\mathrm{CH}_{2}^{+} / \mathrm{C}^{+}$yields enabled fragmentation control from the dication, the opposite alignment dependence of the yields of the $\mathrm{C}_{2} \mathrm{H}^{+} / \mathrm{H}$ channel and the $\mathrm{C}_{2} \mathrm{H}_{2}^{+}$ ion enables channel-selective fragmentation control from 
the singly charged ion. This further highlights the potential of molecular alignment as a versatile tool for controlling fragmentation reactions.

In conclusion, we have introduced molecular alignment as a tool to control the yield of molecular fragmentations on the few-femtosecond time scale. We have achieved for the first time channel selectivity in different fragmentation reactions of a polyatomic molecule initiated by a strong laser field. The method exploits the presence of different ionization channels and the different angular dependence of the ionization probability from inner and outer valence orbitals for controlling the population of dissociative excited electronic surfaces associated with molecular reaction dynamics along specific pathways toward a desired set of fragment ions. We expect that fragmentation control by molecular alignment is applicable to a large class of polyatomic molecules, provided that they can be aligned and feature a distinctively different angular ionization probability from outer and inner valence orbitals.

This work was financed by the Austrian Science Fund (FWF) (Grants No. P21463-N22, No. P25615-N27, No. V193-N16, and No. I274-N16), by a SIRG grant from the ERC (project CyFi), and by a grant from the National Natural Science Foundation of China (No. 11074098). K. D. -D. acknowledges funding by the Max-Planck Research School for Advanced Photon Science.

*Corresponding author. markus.kitzler@tuwien.ac.at

[1] M. Shapiro and P. Brumer, Principles of Quantum Control of Molecular Processes (Wiley, New York, 2003).

[2] M. F. Kling, P. von den Hoff, I. Znakovskaya, and R. de Vivie-Riedle, Phys. Chem. Chem. Phys. 15, 9448 (2013).

[3] M. Kremer, B. Fischer, B. Feuerstein, V. L. B. de Jesus, V. Sharma, C. Hofrichter, A. Rudenko, U. Thumm, C. Schröter, R. Moshammer et al., Phys. Rev. Lett. 103, 213003 (2009).

[4] B. Fischer, M. Kremer, T. Pfeifer, B. Feuerstein, V. Sharma, U. Thumm, C. D. Schröter, R. Moshammer, and J. Ullrich, Phys. Rev. Lett. 105, 223001 (2010).

[5] Y. Liu, X. Liu, Y. Deng, C. Wu, H. Jiang, and Q. Gong, Phys. Rev. Lett. 106, 073004 (2011).

[6] T. Rathje, A. M. Sayler, S. Zeng, P. Wustelt, H. Figger, B. D. Esry, and G. G. Paulus, Phys. Rev. Lett. 111, 093002 (2013).

[7] X. Xie et al., Phys. Rev. Lett. 109, 243001 (2012).

[8] D. Pavičić, K. F. Lee, D. M. Rayner, P. B. Corkum, and D. M. Villeneuve, Phys. Rev. Lett. 98, 243001 (2007).

[9] A. Jaron-Becker, IEEE J. Sel. Top. Quantum Electron. 18, 105 (2012).

[10] I. V. Litvinyuk, K. F. Lee, P. W. Dooley, D. M. Rayner, D. M. Villeneuve, and P. B. Corkum, Phys. Rev. Lett. 90, 233003 (2003).
[11] I. Thomann, R. Lock, V. Sharma, E. Gagnon, S. T. Pratt, H. C. Kapteyn, M. M. Murnane, and W. Li, J. Phys. Chem. A 112, 9382 (2008).

[12] T. K. Kjeldsen, C.Z. Bisgaard, L.B. Madsen, and H. Stapelfeldt, Phys. Rev. A 71, 013418 (2005).

[13] J. L. Hansen, L. Holmegaard, J. H. Nielsen, H. Stapelfeldt, D. Dimitrovski, and L. B. Madsen, J. Phys. B 45, 015101 (2012).

[14] H. Stapelfeldt and T. Seideman, Rev. Mod. Phys. 75, 543 (2003).

[15] A. M. Sayler, T. Rathje, W. Müller, K. Rühle, R. Kienberger, and G. G. Paulus, Opt. Lett. 36, 1 (2011).

[16] R. Dörner, V. Mergel, O. Jagutzki, L. Spielberger, J. Ullrich, R. Moshammer, and H. Schmidt-Böcking, Phys. Rep. 330, 95 (2000).

[17] L. Zhang et al., J. Phys. B 45, 085603 (2012).

[18] X. Xie et al., Phys. Rev. Lett. 108, 193004 (2012).

[19] A. S. Alnaser, X. M. Tong, T. Osipov, S. Voss, C. M. Maharjan, B. Shan, Z. Chang, and C. L. Cocke, Phys. Rev. A 70, 23413 (2004).

[20] J. Ortigoso, M. Rodriguez, M. Gupta, and B. Friedrich, J. Chem. Phys. 110, 3870 (1999).

[21] M. Swart, P. T. van Duijnen, and J. G. Snijders, J. Mol. Struct. 458, 11 (1998).

[22] P. Linstrom and W. Mallard, http://webbook.nist.gov.

[23] A. Talebpour, A. Bandrauk, J. Yang, and S. Chin, Chem. Phys. Lett. 313, 789 (1999).

[24] B. K. McFarland, J. P. Farrell, P. H. Bucksbaum, and M. Gühr, Science 322, 1232 (2008).

[25] H. Akagi, T. Otobe, A. Staudte, A. Shiner, F. Turner, R. Dörner, D. M. Villeneuve, and P. B. Corkum, Science 325, 1364 (2009).

[26] P. Hoff, I. Znakovskaya, S. Zherebtsov, M. Kling, and R. Vivie-Riedle, Appl. Phys. B 98, 659 (2010).

[27] Z. Wu, C. Wu, X. Liu, Y. Deng, Q. Gong, D. Song, and H. $\mathrm{Su}$, J. Phys. Chem. A 114, 6751 (2010).

[28] A. E. Boguslavskiy, J. Mikosch, A. Gijsbertsen, M. Spanner, S. Patchkovskii, N. Gador, M. J. J. Vrakking, and A. Stolow, Science 335, 1336 (2012).

[29] J. Wu, L. P. H. Schmidt, M. Kunitski, M. Meckel, S. Voss, H. Sann, H. Kim, T. Jahnke, A. Czasch, and R. Dörner, Phys. Rev. Lett. 108, 183001 (2012).

[30] C. Wu, H. Zhang, H. Yang, Q. Gong, D. Song, and H. Su, Phys. Rev. A 83, 033410 (2011).

[31] J. Mikosch, A. E. Boguslavskiy, I. Wilkinson, M. Spanner, S. Patchkovskii, and A. Stolow, Phys. Rev. Lett. 110, 023004 (2013).

[32] S. Weber, M. Oppermann, M. Ivanov, and J. Marangos, J. Mod. Opt. 60, 1379 (2013).

[33] A. Castro, H. Appel, M. Oliveira, C. A. Rozzi, X. Andrade, F. Lorenzen, M. A. L. Marques, E. K. U. Gross, and A. Rubio, Phys. Status Solidi B 243, 2465 (2006).

[34] C. Cornaggia and P. Hering, Phys. Rev. A 62, 023403 (2000).

[35] T. S. Zyubina, Y. A. Dyakov, S. H. Lin, A. D. Bandrauk, and A. M. Mebel, J. Chem. Phys. 123, 134320 (2005).

[36] M. Hochlaf, S. Taylor, and J. H. D. Eland, J. Chem. Phys. 125, 214301 (2006). 